\title{
Safety Measures During Cholecystectomy: Results of a Nationwide Survey
}

\author{
K. T. Buddingh $\cdot$ H. S. Hofker • \\ H. O. ten Cate Hoedemaker - G. M. van Dam • \\ R. J. Ploeg $\cdot$ V. B. Nieuwenhuijs
}

Published online: 29 March 2011

(C) The Author(s) 2011. This article is published with open access at Springerlink.com

\begin{abstract}
Background This study aimed to identify safety measures practiced by Dutch surgeons during laparoscopic cholecystectomy.

Method An electronic questionnaire was sent to all members of the Dutch Society of Surgery with a registered e-mail address.

Results The response rate was $40.4 \%$ and 453 responses were analyzed. The distribution of the respondents with regard to type of hospital was similar to that in the general population of Dutch surgeons. The critical view of safety (CVS) technique is used by $97.6 \%$ of the surgeons. It is documented by $92.6 \%$, mostly in the operation report $(80.0 \%)$, but often augmented by photography $(42.7 \%)$ or video (30.2\%). If the CVS is not obtained, $50.9 \%$ of surgeons convert to the open approach, $39.1 \%$ continue laparoscopically, and $10.0 \%$ perform additional imaging studies. Of Dutch surgeons, 53.2\% never perform intraoperative cholangiography (IOC), $41.3 \%$ perform it incidentally, and only $2.6 \%$ perform it routinely. A total of 105 bile duct injuries (BDIs) were reported in 14,387 cholecystectomies $(0.73 \%)$. The self-reported major BDI rate (involving the common bile duct) was $0.13 \%$, but these figures need to be confirmed in other studies.

Conclusion The CVS approach in laparoscopic cholecystectomy is embraced by virtually all Dutch surgeons. The course of action when CVS is not obtained varies. IOC seems to be an endangered skill as over half the Dutch
\end{abstract}

K. T. Buddingh - H. S. Hofker - H. O. ten Cate Hoedemaker . G. M. van Dam - R. J. Ploeg - V. B. Nieuwenhuijs $(\bowtie)$ Department of Surgery, University Medical Centre Groningen, P. O. Box 30.001, 9700 RB Groningen, The Netherlands e-mail: v.b.nieuwenhuijs@chir.umcg.nl surgeons never perform it and the rest perform it only incidentally.
Abbreviations
BDI Bile duct injury
CVS Critical view of safety
IOC Intraoperative cholangiography
CBD Common bile duct

\section{Introduction}

After laparoscopic cholecystectomy was introduced in the early 1990s, an increase in the number of bile duct injuries (BDIs) was noted [1]. A BDI has serious medical, financial, and medicolegal consequences for patients and health-care professionals [2-4]. Subsequently, additional patient safety interventions were implemented.

A major step toward safe cholecystectomy was the description of the "critical view of safety" (CVS) technique by Strasberg in 1995 [5]. The CVS technique is advocated by virtually all recent guidelines and expert commentaries [6-9]. The Dutch Society of Surgery issued a best-practice guideline in 2005 endorsing the CVS technique [10]. According to the guideline, CVS is achieved once one third of the gallbladder is dissected off the liver, and the presumed cystic duct and artery are the only structures running from the gallbladder to the hepatoduodenal ligament. The guidelines were promoted through publication and presentation at national conferences; all Dutch surgeons are expected to follow them.

Another safety intervention is intraoperative cholangiography (IOC). Population-based analyses have shown a reduction in major BDI by $25-39 \%$ when IOC is performed 
[11-13]. The guidelines of the Dutch Society of Surgery currently do not recommend routine IOC.

It has been suggested that the incidence of BDIs in the Netherlands is higher than in other countries [2]. Numerous papers have been published on preventive measures to take during cholecystectomy, but it remains unclear which safety measures are actually being employed by Dutch surgeons. This study aimed to identify practice of and opinions on the CVS technique and IOC.

\section{Methods}

An electronic questionnaire was composed by a panel of five abdominal surgeons and a medical psychologist (Appendix). The survey was completely anonymous. E-mail addresses were obtained of all members of the Dutch Society of Surgery, including surgical trainees, and the electronic questionnaire was sent to all addresses. Two weeks after the first e-mail, one reminder was sent. The study closed for recruitment 2 weeks later.

Hospitals in the Netherlands may be classified as "university teaching" (tertiary referral, specialist training, and medical research), "nonuniversity teaching" (general hospitals licensed to train surgical trainees), or "nonteaching" (general hospitals that do not train surgical trainees). Once deemed sufficiently qualified, surgical trainees may perform cholecystectomies without supervision of a consultant present in operating theater.

Statistical analysis was performed with SPPS ver. 16.0 for Windows (SPPS Inc., Chicago, IL, USA). Descriptive statistics were used to portray the responses. The accumulated number of cholecystectomies performed by the groups of surgeons was calculated using the median of the self-reported range of cholecystectomies (i.e., 17 for the range of 10-25). In case of $>50$ cholecystectomies per year, the arbitrarily chosen number of 60 was used. $\chi^{2}$ tests were used to compare the incidence of BDIs in different groups. A $P<0.05$ was considered significant.

\section{Results}

The electronic survey was successfully delivered to 1206 addresses. There was a $40.4 \%$ (487/1206) response rate. Thirty-four surgeons indicated that they no longer performed cholecystectomies. Thus, 453 questionnaires were included for analysis.

Clinical profile

The clinical profile of the respondents is shown in Table 1 . Most respondents were abdominal or hepatobiliary surgeons
(31.3\%), followed by surgeons of other subspecialties $(28.9 \%)$ and surgical trainees $(23.8 \%)$. The majority of respondents worked in nonuniversity teaching hospitals $(56.7 \%)$, followed by university hospitals $(22.7 \%)$ and nonteaching hospitals (20.5\%). This closely resembles the general distribution in the Netherlands [14]. The total number of estimated cholecystectomies in the last 12 months was 14,387

\section{Operative technique}

The CVS technique was used by $97.6 \%$ of the respondents. It was documented by $91.6 \%$, usually in the operation notes. Photographs of the CVS were stored by $42.7 \%$ of surgeons and video images by $30.1 \%$. If the CVS cannot be obtained, $50.9 \%$ opt for conversion to open surgery, $39.1 \%$ continue laparoscopically, and $10.0 \%$ perform additional imaging studies.

Intraoperative imaging studies

More than half of the surgeons (53.2\%) never perform IOC. The remainder use it incidentally $(<5 \%)$. Only $2.6 \%$ of the surgeons perform IOC routinely ( $>80 \%$ of cholecystectomies). Indications for IOC according to the surgeons were suspected BDI $(53.0 \%)$, unclear anatomy $(46.6 \%)$, and suspected common bile duct (CBD) stones (38.0\%). Laparoscopic ultrasound was used by $2.1 \%$ of the surgeons.

Bile duct injuries

Of the respondents, $20.3 \%$ had experienced one or more cases of BDI in the past 12 months. These injuries involved the cystic and Luschkan ducts (type A injuries according to

Table 1 Clinical profile of the respondents

\begin{tabular}{lr}
\hline & \multicolumn{1}{l}{$N$} \\
\hline Differentiation & \\
Surgeon, abdominal or hepatobiliary & $142(31.3 \%)$ \\
Surgeon, other subspecialty & $131(28.9 \%)$ \\
General surgeon/fellow & $49(15.9 \%)$ \\
Surgical trainee & $108(23.8 \%)$ \\
Type of hospital & \\
University hospital & $103(22.7 \%)$ \\
Teaching hospital & $257(56.7 \%)$ \\
Nonteaching hospital & $91(20.5 \%)$ \\
No. of cholecystectomies in past 12 months & \\
$<10$ & $60(13.2 \%)$ \\
$10-25$ & $117(25.8 \%)$ \\
$26-50$ & $194(42.8 \%)$ \\
$>50$ & $82(18.1 \%)$ \\
\hline
\end{tabular}


the Amsterdam criteria [15]) in $77.2 \%$ of cases and the CBD (nine cases of type B and ten type D) in $18.1 \%$ (Table 2). The self-reported BDI rate was $105 / 14,387=$ $0.73 \%$. The self-reported rate of major BDI (i.e., involving the CBD) was $19 / 14,387=0.13 \%$.

The rate of self-reported major BDI was not correlated with the level of training of the surgeon, the course of action if CVS could not be obtained, or the use of IOC (Table 3). There was a nonsignicantly lower rate of major BDI in university hospitals $(P=0.098)$ and a higher rate in the group of surgeons who perform fewer than 10 cholecystectomies per year $(P=0.082)$. These figures are based upon self-reporting and were not corrected for the indication for performing the cholecystectomy, i.e., cholecystitis or biliary colic.

\section{Opinions on IOC}

IOC was regarded as cumbersome by $39.0 \%$ of the surgeons (Table 4). Most surgeons (77.5\%) assume IOC will take $10-30 \mathrm{~min}$. Around one third of the surgeons think IOC reduces the risk of major BDI, one third does not, and one third does not know. A large majority (92.9\%) of the surgeons feel that IOC should not be performed routinely.

\section{Trainees}

All trainees reported use of the CVS technique versus $96.8 \%$ of other surgeons $(P=0.074)$. There were more trainees who never performed IOC than other surgeons $(72.2 \%$ vs. $47.2 \%, P=0.002$ in linear-by-linear association).

\section{Discussion}

The current study is an inventory of safety measures during cholecystectomy in a broad population of general surgeons and trainees in the Netherlands. The response rate was fair with $40.4 \%$, allowing a comparison with a similar survey in the United States [16] and a survey among British and Irish upper-GI surgeons [17]. The distribution of the respondents with regard to type of hospital resembled the general distribution in the Netherlands. The self-reported number of cholecystectomies performed yearly represents about $60 \%$ of the 24,000 performed yearly [18]. The survey therefore provides a reliable representation of the general Dutch practice.

The critical view of safety (CVS) was found to be widely accepted in Dutch practice: $97.6 \%$ of the respondents use this technique. Reviewing 13 Dutch cholecystectomy protocols in 2008, Wauben et al. [19] found that only one explicitly incorporated the use of CVS. It seems
Table 2 Operative technique and imaging

\begin{tabular}{|c|c|}
\hline & $N$ \\
\hline \multicolumn{2}{|l|}{ CVS technique used } \\
\hline Yes & $442(97.6 \%)$ \\
\hline No & $11(2.4 \%)$ \\
\hline \multicolumn{2}{|l|}{ CVS documented } \\
\hline Yes & $405(91.6 \%)$ \\
\hline No & $37(8.4 \%)$ \\
\hline N/A & 11 \\
\hline \multicolumn{2}{|l|}{ CVS documented by ${ }^{a}$} \\
\hline Operation notes & $324(80.0 \%)$ \\
\hline Photograph & $173(42.7 \%)$ \\
\hline Video & $122(30.1 \%)$ \\
\hline N/A & 48 \\
\hline \multicolumn{2}{|l|}{ What course when CVS is not obtained } \\
\hline Usually continue laparoscopically & $165(39.1 \%)$ \\
\hline Usually convert to open & $225(50.9 \%)$ \\
\hline Usually additional imaging studies & $44(10.0 \%)$ \\
\hline N/A & 11 \\
\hline \multicolumn{2}{|l|}{ IOC performed } \\
\hline Never & $241(53.2 \%)$ \\
\hline$<5 \%$ & $187(41.3 \%)$ \\
\hline $5-20 \%$ & $8(1.8 \%)$ \\
\hline $21-80 \%$ & $5(1.1 \%)$ \\
\hline$>80 \%$ & $12(2.6 \%)$ \\
\hline \multicolumn{2}{|l|}{ Indications for $\mathrm{IOC}^{\mathrm{a}}$} \\
\hline Routine & $17(3.8 \%)$ \\
\hline Suspected CBD stones & $172(38.0 \%)$ \\
\hline Unclear anatomy & $211(46.6 \%)$ \\
\hline Suspected BDI & $240(53.0 \%)$ \\
\hline Other & $54(11.9 \%)$ \\
\hline \multicolumn{2}{|l|}{ Laparoscopic ultrasound performed } \\
\hline Never & $443(97.8 \%)$ \\
\hline$<5 \%$ & $7(1.5 \%)$ \\
\hline $5-20 \%$ & $2(0.4 \%)$ \\
\hline $21-80 \%$ & 0 \\
\hline$>80 \%$ & $1(0.2 \%)$ \\
\hline \multicolumn{2}{|l|}{$\mathrm{BDI}$ in the past 12 months } \\
\hline None & $361(79.7 \%)$ \\
\hline 1 & $79(17.4 \%)$ \\
\hline 2 & $13(2.9 \%)$ \\
\hline$>2$ & 0 \\
\hline \multicolumn{2}{|l|}{ Types of BDI } \\
\hline Cystic stump leak & 53 \\
\hline Luschkan duct leak & 28 \\
\hline CBD leak & 9 \\
\hline CBD transsection & 10 \\
\hline Other & 5 \\
\hline
\end{tabular}

$C V S$ critical view of safety, $I O C$ intraoperative cholangiography, $C B D$ common bile duct, $B D I$ bile duct injury, N/A not applicable

${ }^{\text {a }}$ Multiple answers were possible 
Table 3 Factors associated with major BDI (i.e., involving the $\mathrm{CBD}$ )
$B D I$ bile duct injury, $I O C$ intraoperative cholangiography, $C V S$ critical view of safety

a Calculated by multiplying the number of surgeons by the median of the reported range of cholecystectomies performed yearly, and by 60 for those who reported to perform more than 50 per year

b These constituted nine type B injuries and ten type $\mathrm{D}$ injuries [15]

${ }^{c}$ For the surgeons who indicated that they used the CVS technique

\begin{tabular}{|c|c|c|c|c|}
\hline & $\begin{array}{l}\text { No. of } \\
\text { surgeons }\end{array}$ & $\begin{array}{l}\text { Accumulated No. of } \\
\text { cholecystectomies }^{\mathrm{a}}\end{array}$ & $\begin{array}{l}\text { Major } \\
\text { BDI }^{\mathrm{b}}\end{array}$ & $P$ \\
\hline Differentiation & & & & 0.621 \\
\hline Abdominal/HPB surgeon & 142 & 5267 & $6(0.11 \%)$ & \\
\hline Other subspecialty & 131 & 3786 & $7(0.18 \%)$ & \\
\hline General surgeon / fellow & 49 & 2580 & $4(0.16 \%)$ & \\
\hline Surgical trainee & 108 & 2754 & $2(0.07 \%)$ & \\
\hline Type of hospital & & & & 0.098 \\
\hline University teaching & 103 & 2005 & 0 & \\
\hline Nonuniversity teaching & 257 & 8679 & $11(0.13 \%)$ & \\
\hline Nonteaching & 91 & 3703 & $8(0.22 \%)$ & \\
\hline No. of cholecystectomies in past 12 months & & & & 0.082 \\
\hline$<10$ & 60 & 300 & $2(0.67 \%)$ & \\
\hline $10-25$ & 117 & 1989 & $2(0.10 \%)$ & \\
\hline $26-50$ & 194 & 7178 & $9(0.13 \%)$ & \\
\hline$>50$ & 82 & 4920 & $6(0.12 \%)$ & \\
\hline What course when CVS is not obtained ${ }^{\mathbf{c}}$ & & & & 0.350 \\
\hline Usually continue laparoscopically & 165 & 5139 & $8(0.16 \%)$ & \\
\hline Usually convert to open & 225 & 7291 & $11(0.15 \%)$ & \\
\hline Usually additional imaging & 44 & 1369 & 0 & \\
\hline IOC performed & & & & 0.505 \\
\hline Never & 241 & 7495 & $12(0.16 \%)$ & \\
\hline$<5 \%$ & 187 & 6342 & $6(0.09 \%)$ & \\
\hline $5-20 \%$ & 8 & 218 & $1(0.46 \%)$ & \\
\hline $21-80 \%$ & 5 & 136 & 0 & \\
\hline$>80 \%$ & 12 & 196 & 0 & \\
\hline
\end{tabular}

that although protocols need to be updated in some hospitals, the CVS is widely accepted in the Netherlands as the gold standard. The implementation of the most important safety measure to prevent bile duct injury (BDI) can thus be considered highly successful and is praiseworthy. In a similar survey by Sanjay et al. [17], 82\% of the British and Irish upper-GI surgeons advocated the CVS technique. It is unknown how often this technique is actually practiced by British general surgeons or how well institutionalized the CVS technique is in other countries.

Documentation of the CVS in the operation notes is done by $80.0 \%$ of surgeons, and augmented by a majority by video or photographs. The course of action when CVS is not obtained varies. Although the nature of the question does not address some of the nuances in difficult cholecystectomies, a divergent strategic approach of the surgeons is illustrated. Timely conversion in case of uncertain anatomy is seen by many surgeons as an important safety measure. However, as the open approach is increasingly reserved for "difficult cases" and experience with the open technique diminishes, there are increasing reports of BDI occurring after conversion [20, 21]. Depending on the experience of the surgeon, other alternatives such as laparoscopic subtotal cholecystectomy may in some cases be safer than conversion. In a Dutch series of 1509 patients, experienced laparoscopy surgeons were four times less likely to convert than less experienced laparoscopy surgeons $(3.6 \%$ vs. $15.6 \%)$ [22]. The conversion rate in the Netherlands varies; up to $18 \%$ has been reported [23]. These papers, like most, do not assess whether CVS was achieved.

Intraoperative cholangiography (IOC) is very seldom performed in the Netherlands; $53.1 \%$ of surgeons never use it and $41.3 \%$ perform it incidentally ( $<5 \%$ of cholecystectomies). This contrasts with the practice in the US and the UK, where over $25 \%$ of surgeons routinely perform IOC and there are few surgeons who never apply it $[16,17]$. In Australia, IOC is performed in over $60 \%$ of cholecystectomies [12]. Despite the fact that many Dutch surgeons feel that suspected common bile duct (CBD) stones, unclear anatomy, and suspected bile duct injury (BDI) are indications for IOC, in clinical practice they only rarely apply it.

Approximately one third of the responding surgeons believed that IOC reduces the risk of BDI, one third did not, and one third indicated that they did not know. Opinions were divided on whether IOC was a cumbersome procedure and how much time it consumes. The great 
Table 4 Opinions on IOC

\begin{tabular}{lc}
\hline & $N$ \\
\hline Performing IOC is cumbersome & \\
Usually & $74(16.3 \%)$ \\
More often than not & $103(22.7 \%)$ \\
Sometimes & $127(28.0 \%)$ \\
Usually not & $114(25.2 \%)$ \\
Missing & $35(7.7 \%)$ \\
How long does IOC take & \\
$<10$ min & $30(6.7 \%)$ \\
$10-20$ min & $179(39.5 \%)$ \\
$20-30$ min & $170(37.5 \%)$ \\
$30-40$ min & $50(11.0 \%)$ \\
$>40$ min & $24(5.3 \%)$ \\
IOC reduces the risk of major BDI & \\
Yes & $134(29.6 \%)$ \\
No & $153(33.8 \%)$ \\
Don't know & $136(36.6 \%)$ \\
IOC should be performed routinely & \\
Not & $421(92.9 \%)$ \\
In all teaching hospitals & $21(4.6 \%)$ \\
In all hospitals & $11(2.4 \%)$ \\
\hline
\end{tabular}

IOC intraoperative cholangiography, $B D I$ bile duct injury, $I O C$ intraoperative cholangiography

majority of surgeons $(93 \%)$ believe that IOC should not be routinely practiced. This is remarkable as many of these surgeons believed that IOC reduces the risk of BDI. Apparently, the arguments against routine IOC are thought to outweigh the benefits. Additionally, Dutch insurance companies currently do not reimburse the surgeon for performing IOC. The guidelines currently do not advise selective or routine IOC, and this is reflected in the daily practice of Dutch surgeons. Although the discussion on whether to perform IOC routinely or selectively is far from closed, it seems undesirable that surgeons would lose the skill of IOC altogether. We advocate a low threshold for IOC, especially in complicated biliary disease such as cholangitis and pancreatitis, and certainly in cases of unclear anatomy. An attitude change may be necessary in order for Dutch surgeons to apply IOC more frequently as an investment in patient safety.

The self-reported major BDI rate (i.e., involving the CBD) was $0.13 \%$. This is much lower than the figures mentioned in the literature and similar to the rate observed before the laparoscopic era $[11,24]$. Caution is necessary interpreting this figure as a survey such as this is not the optimal tool to assess the occurrence of complications. No evidence could be found in the literature on the validity of self-reported complications by surgeons. Further research is needed to confirm this low complication rate. The most important limitation of this study is that it relies on selfreporting. It cannot be confirmed that the surgeons use the techniques that they report to use and to what extent. However, the results are certainly of interest as they reflect opinions on and the acceptance of safety measures during cholecystectomy.

In summary, our survey provides insight into safety precautions taken by Dutch surgeons to prevent BDI during cholecystectomy. The CVS approach is embraced by virtually all Dutch surgeons. When CVS is not obtained, different approaches are used, with half of the surgeons choosing to convert. IOC seems to be an endangered skill as over half the Dutch surgeons never perform it and the rest do so only incidentally. Although one may argue as to whether IOC should be performed routinely or selectively, it seems an undesirable development that surgeons would lose the skill of IOC altogether.

Acknowledgments We thank all the Dutch surgeons who took the time to participate in this survey. We are also indebted to Dr. J. Niesing for his help with the development of the questionnaire.

Conflict of interest The authors have no conflicts of interest or financial ties to disclose.

Open Access This article is distributed under the terms of the Creative Commons Attribution Noncommercial License which permits any noncommercial use, distribution, and reproduction in any medium, provided the original author(s) and source are credited.

\section{Appendix: Surgeon survey (translated from Dutch)}

Part A: Profile

1. What is your differentiation?
a. Abdominal or hepatobiliary surgeon
b. Otherwise differentiated surgeon
c. General surgeon
d. Fellow
e. Surgical trainee

2. What type of hospital do you work in?
a. University medical centre
b. Teaching hospital
c. Non-teaching hospital

3. How many cholecystectomies have you performed or supervised in the past 12 months?
a. $<10$
b. $10-25$
c. $25-50$
d. $>50$ 
Part B: Operative technique

4. Do you use the "Critical View of Safety" technique?
a. Yes
b. No

5. Do you document the "Critical View of Safety"?
a. Yes
b. No
c. Not applicable

6. How do you usually register the "Critical View of Safety"? (Multiple answers possible)
a. Not
b. Operation report
c. Photograph
d. Video

7. What do you do when you cannot achieve the "Critical View of Safety"?
a. Usually continue laparoscopically
b. Usually convert
c. Usually perform additional imaging studies

8. How often do you perform intraoperative cholangiography during cholecystectomy?
a. Never
b. $1-5 \%$
c. $5-20 \%$
d. $20-80 \%$
e. $>80 \%$

9. What are indications for you to perform intraoperative cholangiography?
a. Routinely during every cholecystectomy
b. Suspected common bile duct stones
c. Unclear anatomy
d. Suspected bile duct injury
e. Other

10. How often do you perform laparoscopic ultrasound for intraoperative visualization of bile ducts?
a. Never
b. $1-5 \%$
c. $5-20 \%$
d. $20-80 \%$
e. $>80 \%$

11. How often, in the past 12 months, was one of your cholecystectomies complicated by bile duct injury?
a. 0
b. 1
c. 2
d. 3
e. $>3$

12. If there was a bile duct injury, what type was it?
a. Cystic stump leakage
b. Luschkan duct
c. Common bile duct leakage
d. Common bile duct transection
e. Other injuries

Part C: Opinions on intraoperative cholangiography

13. Performing intraoperative cholangiography is a cumbersome procedure
a. Usually
b. More often than not
c. Sometimes
d. Usually not

14. By how much time does intraoperative cholangiography prolong the procedure?
a. 1-10 minutes
b. 10-20 minutes
c. 20-30 minutes
d. 30-40 minutes
e. $>40$ minutes

15. Intraoperative cholangiography reduces the risk of major bile duct injury.
a. Agree
b. Disagree
c. Don't know

16. Intraoperative cholangiography should be performed routinely.
a. Not
b. In all teaching hospitals
c. In all hospitals

\section{References}

1. Nuzzo G, Giuliante F, Giovannini I et al (2005) Bile duct injury during laparoscopic cholecystectomy: results of an Italian national survey on 56591 cholecystectomies. Arch Surg 140(10):986-992

2. de Reuver PR, Rauws EA, Bruno MJ et al (2007) Survival in bile duct injury patients after laparoscopic cholecystectomy: a multidisciplinary approach of gastroenterologists, radiologists, and surgeons. Surgery 142(1):1-9

3. de Reuver PR, Sprangers MA, Rauws EA et al (2008) Impact of bile duct injury after laparoscopic cholecystectomy on quality of life: a longitudinal study after multidisciplinary treatment. Endoscopy 40(8):637-643 
4. de Reuver PR, Wind J, Cremers JE et al (2008) Litigation after laparoscopic cholecystectomy: an evaluation of the Dutch arbitration system for medical malpractice. J Am Coll Surg 206(2):328-334

5. Strasberg SM, Hertl M, Soper NJ (1995) An analysis of the problem of biliary injury during laparoscopic cholecystectomy. J Am Coll Surg 180(1):101-125

6. Callery MP (2006) Avoiding biliary injury during laparoscopic cholecystectomy: technical considerations. Surg Endosc 20(11): $1654-1658$

7. Elakkary E, Ching K, Jacobs MJ (2006) Spiral cystic duct: beware. JSLS 10(4):514-516

8. Strasberg SM (2002) Avoidance of biliary injury during laparoscopic cholecystectomy. J Hepatobiliary Pancreat Surg 9(5): $543-547$

9. Overby DW, Apelgren KN, Richardson W et al (2010) SAGES guidelines for the clinical application of laparoscopic biliary tract surgery. Surg Endosc 24(10):2368-2386

10. Lange JF, Stassen LP (2006) Best practice: De techniek van de laparoscopische cholecystectomie. Critical View of Safety (CVS), Werkgroep Endoscopische Chirurgie van de Nederlandse Vereniging voor Heelkunde. http://www.artsennet.nl/Richtlijnen/ Richtlijn/Laparoscopische-Cholecystectomie.htm. Accessed Oct 2010

11. Flum DR, Dellinger EP, Cheadle A et al (2003) Intraoperative cholangiography and risk of common bile duct injury during cholecystectomy. JAMA 289(13):1639-1644

12. Hobbs MS, Mai Q, Knuiman MW et al (2006) Surgeon experience and trends in intraoperative complications in laparoscopic cholecystectomy. Br J Surg 93(7):844-853

13. Waage A, Nilsson M (2006) Iatrogenic bile duct injury: a population-based study of 152776 cholecystectomies in the Swedish Inpatient Registry. Arch Surg 141(12):1207-1213
14. Dutch Society of Surgery Yearbook 2010

15. Bergman JJ, van den Brink GR, Rauws EA et al (1996) Treatment of bile duct lesions after laparoscopic cholecystectomy. Gut 38(1):141-147

16. Massarweh NN, Devlin A, Elrod JA et al (2008) Surgeon knowledge, behavior, and opinions regarding intraoperative cholangiography. J Am Coll Surg 207(6):821-830

17. Sanjay P, Kulli C, Polignano F et al (2010) Optimal surgical technique, use of intraoperative cholangiography and management of acute gallbladder disease: the results of a nationwide survey in the UK and Ireland. Ann R Coll Surg Engl 92(4):302-306

18. Inspectie voor de Gezondheidzorg (IGZ) (2007) The result counts. Available at http://www.igz.nl/zoeken/document.aspx ?doc=Het_resultaat_telt_2007\&URL=. Accessed Oct 2010

19. Wauben LS, Goossens RH, van Eijk DJ et al (2008) Evaluation of protocol uniformity concerning laparoscopic cholecystectomy in the Netherlands. World J Surg 32(4):613-620

20. Booij KA, de Reuver PR, van Delden OM et al (2009) Conversion has to be learned: bile duct injury following conversion to open cholecystectomy. Ned Tijdschr Geneeskd 153:A296

21. Wolf AS, Nijsse BA, Sokal SM et al (2009) Surgical outcomes of open cholecystectomy in the laparoscopic era. Am J Surg 197(6): 781-784

22. Kortram K, Reinders JS, van Ramshorst B et al (2010) Laparoscopic cholecystectomy for acute cholecystitis should be performed by a laparoscopic surgeon. Surg Endosc 24(9):2206-2209

23. Veen EJ, Bik M, Janssen-Heijnen ML et al (2008) Outcome measurement in laparoscopic cholecystectomy by using a prospective complication registry: results of an audit. Int $\mathbf{J}$ Qual Health Care 20(2):144-151

24. Shea JA, Healey MJ, Berlin JA et al (1996) Mortality and complications associated with laparoscopic cholecystectomy. A metaanalysis. Ann Surg 224(5):609-620 